\title{
IoT-enabled Dependable Co-located Low-cost Sensing for Construction Site Monitoring
}

\author{
Huynh A.D. Nguyen, Lanh V. Nguyen, and Quang P. Ha \\ Faculty of Engineering and Information Technology, \\ University of Technology Sydney, Australia \\ Email: huynhanhduy.nguyen@uts.edu.au, vanlanh.nguyen@uts.edu.au, quang.ha@uts.edu.au
}

\begin{abstract}
-
This paper proposes an IoT-enabled network of low-cost sensors that are co-located for construction site monitoring. The network performance enhancement is achieved via its system dependability in terms of improved availability, integrity, reliability, maintainability, security and safety in real-time monitoring of environment parameters. The sensor motes of various sensing modules form a reliable wireless in-situ cluster for gathering on-site information of air temperature, soil moisture, air pressure, humidity, particulate matters (PM), emissions and weather variables. They are useful for the site management, improving safety and effective operation of construction equipment. The components for the development include inexpensive microcontrollers ESP32 embedded with wireless gateway function and energy-efficient motes featuring cost-effective sensors. Here, the adoption of the dependability concept for collocated sensor motes aims to introduce a level of redundancy to allow for improving fault-tolerance and reliability. Extensive field tests have been conducted in different environments. Experimental results as well as statistical analysis are provided to verify the merits of the proposed approach.
\end{abstract}

Keywords -

Site monitoring; Volatile environment; Wireless sensor motes; Dependability; Reliability

\section{Introduction}

The Internet of Things (IoT) has been applied into various pieces of equipment which are called smart devices, incorporating digital intelligence. However, most of the IoT devices operate indoors with optimized temperatures, relative humidity $(\mathrm{RH})$, dust filter or in well-controlled conditions, that may incur extra budget and resources. The rapid innovation of electronic platforms offers to mitigate these constraints from the availability of commercially low-cost devices. Nevertheless, the quality and performance of these inexpensive components need to be verified and standardized in practical conditions where unpredictable variations or imperfect conditions are unavoidable. The low-cost wireless sensor networks (WSN) adopted for environment-monitoring is an example for addressing this concern. The promising use of IoT-enabled systems operating in the harsh environment has recently attracted researchers in many areas including construction automation. A low-cost sensing system named AIRQino [1] was designed and developed to be as the auxiliary observatory point combining with high-quality reference stations in the Arctic. Cost-effective WSN are also being implemented for military, agriculture, transportation, on land and in marine environment [2], in which instruments may be easily prone to damage. In the construction sector, the IoT-based low-cost WSN technology is still in its infancy [3]. On a construction site, most of the studies focus on critical themes of safety, construction progress monitoring, BIM-related technology, project management, machine and resource management [4], which often consider normal operations with less concern to difficult conditions on a jobsite that may be subject to uncertainty and unpredictability.

Volatility in a jobsite is referred to as varying, unpredictable conditions, such as with varying weather, which may involve drastic changes in construction activities, and cause serious impacts. For example, earthworks usually take place under difficult conditions that have been widely referred to as 3D's, namely Dull, Dirty and Dangerous. In addition being vulnerable to a volatile environment, a construction process may also encounter abnormal disadvantages known as 3H's, i.e. Harsh, Hostile and Hazardous, such as in military or some mining and earthmoving missions [5]. Harsh conditions may degrade the quality of collected data and performance of controllers and the whole system. For reliable and resilient performance in construction processes against environmental variations, disturbances, imperfect conditions or ambient changes, the wireless sensing system should improve system dependability to allow for increased robust, safe and fault tolerant monitoring performance in real time. 
The dependability of an IoT system is recently considered and explained as the ability of system proving justifiably trusted services [6]. A dependable system has typical characteristics such as availability, integrity, reliability, maintainability, security and safety. In costsaving wireless sensing systems, reliability and availability are most critical in order to guarantee the continuous communication, data completeness and power consistency [7]. To increase reliability and availability of the system, and hence, resilience of construction or any industrial process, the downtime from the permanent failure of core controllers should be avoided [8]. In this regard, fault tolerance schemes have been developed in both hardware and software to improve dependability of a management system for solar energy consumption in a smart building [9].

This paper presents the design and development of low-cost wireless sensor systems that are collocated and controlled by our proposed dependable scheme. The availability of power supply is discussed by the combination of Dynamic Energy Conservation (DEC) scheme and the low-power network (ESP-NOW) from low-cost platform ESP32 in Section 2. The developed prototype is validated in laboratory before conducting field tests. Onsite collected data are analyzed by using statistic tools, whereby stochastic regression will be used to impute missing data during online operation. Sections 3 and 4 present the field test results for monitoring construction sites in different environments. Finally, a conclusion for the paper is drawn in Section 5.

\section{Development of low-cost wireless sensor system}

\subsection{Hardware system}

Figure 1 shows the proposed diagram of the dependable sensing system, developed for the low-cost WSN, which comprises two subsystems. The first subsystem is called sensor mote which constitutes four identical sensing modules of which one is operating while the others are in a stand-by status. Each IoT device operates as an autonomous sensor module (ASM) that consists of components such as micro-control unit (MCU), environmental sensors (soil moisture, soil temperature, $\mathrm{RH}$, pressure, particulate matter - PM2.5 and PM10), two battery cells - Panasonic Lithium-Ion 18650 (3.7V-3400mAh/cell), one mini solar PV panel, real-time reading module, boost-buck converter and battery charging modules, all at a low cost, as shown in Table 1.

The second subsystem is called gateway mote or router mote. This device is assigned as the role of an intermediate transceiving point between sensor motes and the server when there is no cloud network in a construction site. The gateway mote is assembled by two redundant ESP-32s connecting each other by the serial communication protocol. One MCU communicates regularly with sensor motes in the local network (ESPNOW) and the other connects to the cloud with Wi-Fi standard $802.11 \mathrm{~b} / \mathrm{g} / \mathrm{n}$. Data packets are sent to the Thingspeak server (https://thingspeak.com) by the hyper transfer text protocol (HTTP).

The printed circuit board (PCB) and main components are protected in a waterproof box (IP68 standard) from the intrusive water and dust at construction sites. Although each sensor module is designed separately, four modules are collocated on the same spots, ensuring redundancy of the sensor mote. Figure 2 shows the prototype with electronic components in a sensor module.

Table 1. Main components of the sensor mote

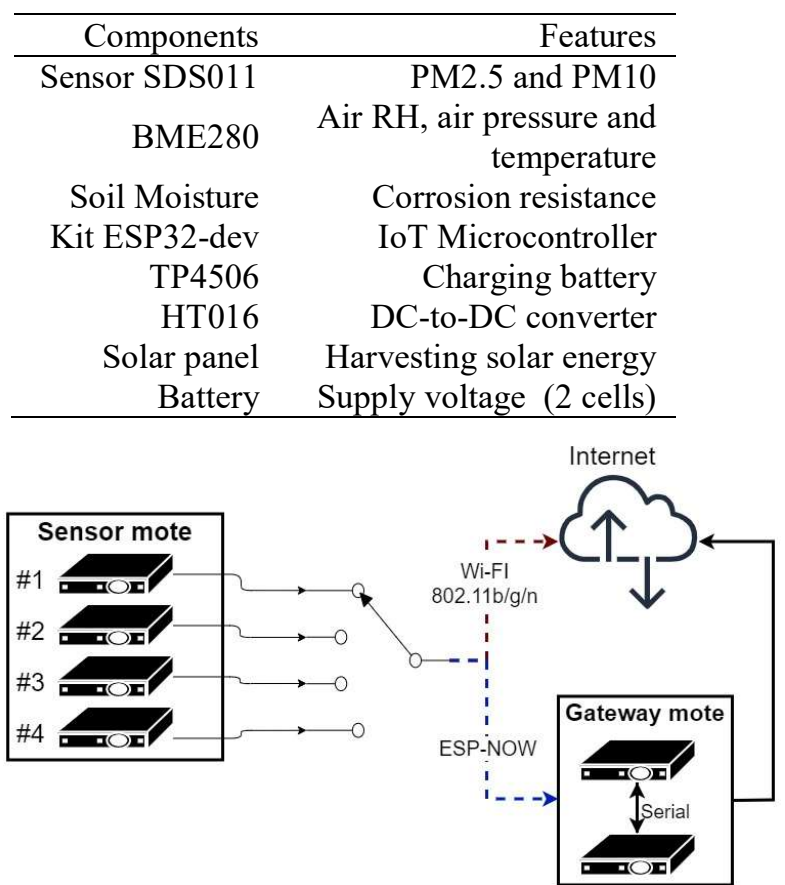

Figure 1. Diagram of the dependable IoT system

\subsection{The microcontroller ESP32 and ESP- NOW communication protocol}

The MCU ESP32, manufactured by Espressif System company, is a low-power System on Chip (SoC) with wireless communication features in both $\mathrm{Wi}-\mathrm{Fi}$ and Bluetooth Low Energy (BLE) protocols. This low-cost platform provides multiple peripherals and various communication types (e.g. PWM, I2C, SPI, RS232, etc.). Thanks to the energy-saving architecture with different power modes and 2-core processing capacity at frequency up to $240 \mathrm{MHz}$, the ESP32 MCU is suitable for cost-saving and real-time solutions [10]. The Wi-Fi protocol in ESP32 offers two IoT functions: Station (ST) 
and Access point (AP), from which the controller can be a member of an available network (e.g. cloud network) or can set up its own local network as a gateway respectively.

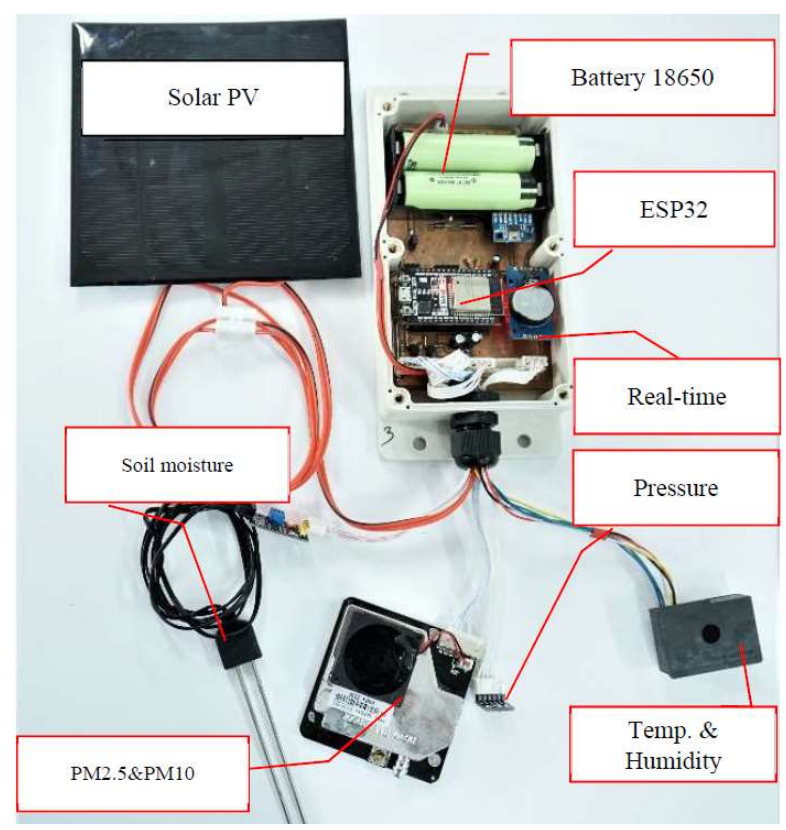

Figure 2. Low-cost sensor mote

\begin{tabular}{|c|c|c|c|c|c|}
\hline MAC Header & Category Code & $\begin{array}{c}\text { Organization } \\
\text { Identifer }\end{array}$ & Random Values & $\begin{array}{c}\text { Vendor Specific } \\
\text { Content }\end{array}$ & FCS \\
\hline 24 bytes & 1 byte & 3 bytes & 4 bytes & $7-255$ bytes & 4 bytes \\
\hline Element ID & Length & $\begin{array}{c}\text { Organization } \\
\text { Identfer }\end{array}$ & Type & Version & Body \\
\hline 1 byte & 1 byte & 3 bytes & 1 byte & 1 byte & $0-250$ bytes \\
\hline
\end{tabular}

Figure 3. Vendor-specific Action Frame and Vendor-specific Content [10]

For the machine-to-machine protocol, the Esprissef wireless ESP-NOW is used, featuring low-power $2.4 \mathrm{GHz}$-radio communication that can connect up to 20 devices without handshake. As shown in the diagram of Figure 3, the data frame of this protocol is encapsulated by a vendor-specific action frame transmitted at the default bit rate of $1 \mathrm{Mbps}$. Moreover, data transceived by ESP-NOW are secured by the cryptography method (CCMP) defined in IEEE 802.11-2012. This method reduces the risk of interference from other devices or networks to assure the dependable feature of the proposed system in terms of network security.

The body of Vendor-specific Content allows the maximum data packet up to 250 bytes which are more than the requirement in this study because the total sensing data are 112 bytes in the proposed system. In order to validate the efficiency of ESP-NOW protocol, the experiment was conducted in the laboratory with the Virtual Bench NI VB-8012 All-in-one instrument, which could capture signals up to $100 \mathrm{MHz}$ to measure and compare the energy consumption and duration for different wireless communication protocols (ESP-NOW vs. Wi-Fi standard).

In the first experiment with ESP-NOW, the current draining for transceiving a 112-byte packet is $324 \mathrm{~mA}$ in $1.6 \mathrm{~ms}$, i.e. a small capacity $\mathrm{C}_{\mathrm{ESP}-\mathrm{NOW}}=0.144 \times 10^{-3} \mathrm{mAh}$. In comparison with sending directly the same packet by Wi-Fi protocol in the second experiment, the expensed capacity is $173 \mathrm{~mA}$ in $1285 \mathrm{~ms}$, equivalent to $\mathrm{C}_{\mathrm{Wi}-\mathrm{Fi}}=$ $61.751 \times 10^{-3} \mathrm{mAh}$. To this end, ESP-NOW communication is selected as the local network connecting with gateway motes due to its energy efficiency in radio communication. Besides, the real discharge current were measured without connecting to network $\left(\mathrm{I}_{\text {measure }}=162 \mathrm{~mA}\right)$ and during the deep-sleep mode $\left(\mathrm{I}_{\text {deep-sleep }}=10 \mathrm{~mA}\right)$ of the module for the later evaluation of power cost in each working cycle of WSN.

\subsection{Dynamic Energy Conservation (DEC) scheme}

We apply the Dynamic Energy Conservation (DEC) scheme based on the saving-energy features of ESP32 platform, which varies the duration of the active-sleep cycle dynamically. Here, the active-sleep cycle of the when sensor module includes (1) the active period counted from the "wake-up" and data collection time; (2) period for connecting the local network and transmitting data successfully; (3) the stand-by (sleep) period from triggering the deep-sleep mode until the next wake-up time.

In environmental monitoring application, the ambient parameters are measured as discrete variables with slow variation (e.g. temperature) to reduce constraints of power resource. Here, the regulation sampling frequency depends on the construction activities in dayshifts or nightshifts to flexibly increase the availability of the system. Next, we will consider the various active-sleep cycles from DEC to optimize the power consumption of this system.

The average discharge current in one cycle is:

$$
I_{\text {cycle }}=\frac{\sum_{i=1}^{3} m_{i} I_{i}}{\sum_{i=1}^{3} m_{i}},
$$

where $m_{i}$ and $I_{i}(i=1,2,3)$ is the period and discharge current according to the mode $i^{\text {th }}$ as listed in Table 3 . In this study, the time to initiate sensors and wireless connection is at least 30 seconds prior to data measurement and transmission, we choose time for active mode $m_{1}=30 \mathrm{~s}$, whereas the ESP-NOW transmission period for a data packet is very small $\left(m_{2}=1.6 \mathrm{~ms}\right)$ that could be neglected. Hence, the dynamic operation cycles 
will be determined by the sleeping time $\left(m_{3}\right)$. We designed a switching mechanism for alternating the battery usage. One battery is charged by solar energy and the other is responsible for supplying power for the whole module.

Table 3. The active-sleep cycle of the sensor module with discharge current measured in each mode

\begin{tabular}{cccc}
\hline Mode & Action & Period & $\begin{array}{c}\text { Discharge } \\
\text { current }\end{array}$ \\
\hline Active & Measure data & Flexible & $162 \mathrm{~mA}$ \\
Active & Communication & $1.6 \mathrm{~ms}$ & $324 \mathrm{~mA}$ \\
Deep-sleep & Stand-by & Flexible & $10 \mathrm{~mA}$ \\
\hline
\end{tabular}

Due to the back-up design, this mechanism remains the power supply over the whole working duration, whereas it reduces the aging problem of the battery by intermittent charge and discharge. Therefore, the energydischarged calculation will be considered for one battery cell. The expected working time of a battery cell in the sensor module is determined by the average discharge current in one cycle (1) and the battery time calculated as

$$
T_{\text {bat }}=\frac{C_{\text {bat }}}{I_{\text {cycle }} \cdot n_{\text {cycles }}},
$$

where $T_{b a t}$ (in hours) is the average service-time of the battery being fully charged; $I_{c y c l e}$ is the average discharge current in one cycle; $n_{\text {cycles }}$ is the number of cycles per hour according to sleeping time, and $C_{\mathrm{bat}}$ is the capacitance discharge current. In our application, $C_{\text {bat }}=$ $3000 \mathrm{~mA}$ from fully-charged to cut-off point (from $4.2 \mathrm{~V}$ to $3.0 \mathrm{~V}$ respectively) with Lithium-Ion NCR18650B battery [11].

From the estimated time listed in Table 4, the larger sleeping period the longer the battery service time. However, the over-extending idle time will reduce the temporal resolution of data, which may cause unreliable analysis results and decisions. This requires further improvement from dependable algorithms [12].

Table 4 . The estimated battery service-time with fixed value of active mode $\left(m_{1}+m_{2} \approx 30 \mathrm{~s} ; \mathrm{T}_{\text {total }}=m_{1}+m_{2}+m_{3}\right)$

\begin{tabular}{ccccc}
\hline $\begin{array}{c}\text { Sleeping } \\
\text { period }\end{array}$ & $\begin{array}{c}\text { Cycle time } \\
\left(\mathrm{T}_{\text {total }}-\mathrm{ms}\right)\end{array}$ & $n_{\text {cycles }}$ & $\begin{array}{c}I_{\text {cycle }} \\
(\mathrm{mA})\end{array}$ & $\begin{array}{c}T_{\text {bat }} \\
(\text { hour })\end{array}$ \\
\hline 30 & 60 & 60 & 91.5 & 0.6 \\
60 & 90 & 40 & 64.3 & 1.2 \\
120 & 150 & 24 & 42.6 & 3.1 \\
300 & 330 & 10.9 & 24.8 & 11.5 \\
600 & 660 & 5.5 & 17.7 & 31.6 \\
900 & 930 & 3.9 & 15.3 & 51.6 \\
\hline
\end{tabular}

\section{Field test and results: on-campus construction site}

\subsection{Field testing and data validation}

The first field test was conducted at a construction site in at coordinates $10.0323^{\circ} \mathrm{N}, 105.7682^{\circ} \mathrm{E}$. Due to the safety policy from the contractor, the on-site testing period was conducted in a short period from $26^{\text {th }}$ to $29^{\text {th }}$ October 2019 and the sampling interval is $15 \mathrm{~min} /$ cycle.

The proposed IoT-enabled dependable scheme illustrated in Figure 1 was implemented with four modules (MD1-MD4), and installed at the site as shown in Figure 4. It will control the communication switch to connect gateways or the server to each sensor module consecutively. Hence, data samples increase four times compared to the single wireless sensor as conventional WSN. As a result, the spatial and temporal distributions of environmental parameters on the construction site have been significantly improved.

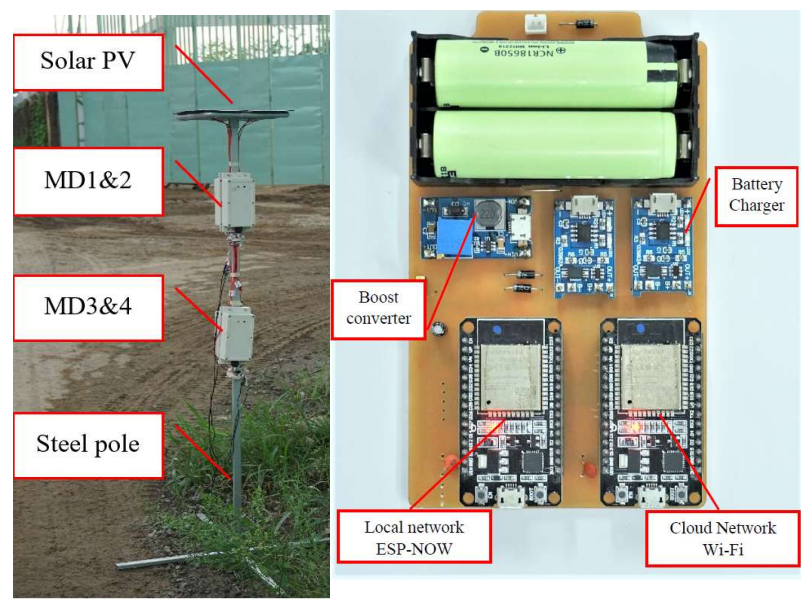

Figure 4. On-site sensor mote (left) and the gateways mote PCB board (right).

The temporal distributions of temperature, RH, PM2.5 and PM10 are illustrated in Figure 5. The profiles of all data show similar trends between collocated modules with small variations. Although some points are affected by noises, they could be removed by filtering out.

To investigate the correlation and linear relationship between the data collected from the low-cost sensor modules, Pearson's correlation coefficient is used

$$
r=\frac{\sum_{i=1}^{n}\left(x_{i}-\bar{x}\right)\left(y_{i}-\bar{y}\right)}{\sqrt{\sum_{i=1}^{n}\left(x_{i}-\bar{x}\right)^{2}} \sqrt{\sum_{i=1}^{n}\left(y_{i}-\bar{y}\right)^{2}}},
$$

where $r$ is the Pearson's correlation coefficient; $x$ and $y$ are the measured values of two sensors; $\bar{x}$ and $\bar{y}$ are means of two datasets and $n$ is the total samples. To evaluate on the deviation between measurements collected from the sensor modules, we use the Mean 
Absolute Error (MAE) and Root Mean Square Error (RMSE) calculated as [13],

$$
\begin{aligned}
M A E & =\frac{1}{n} \sum_{i=1}^{n}\left|x_{i}-y_{i}\right|, \\
R M S E & =\sqrt{\frac{\sum_{i=1}^{n}\left(x_{i}-y_{i}\right)^{2}}{n}} .
\end{aligned}
$$
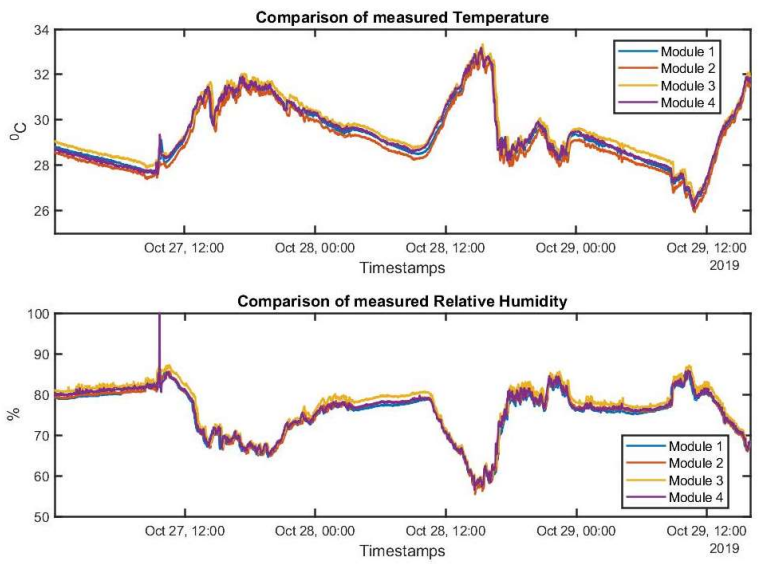

(a) Temperature and relative humidity
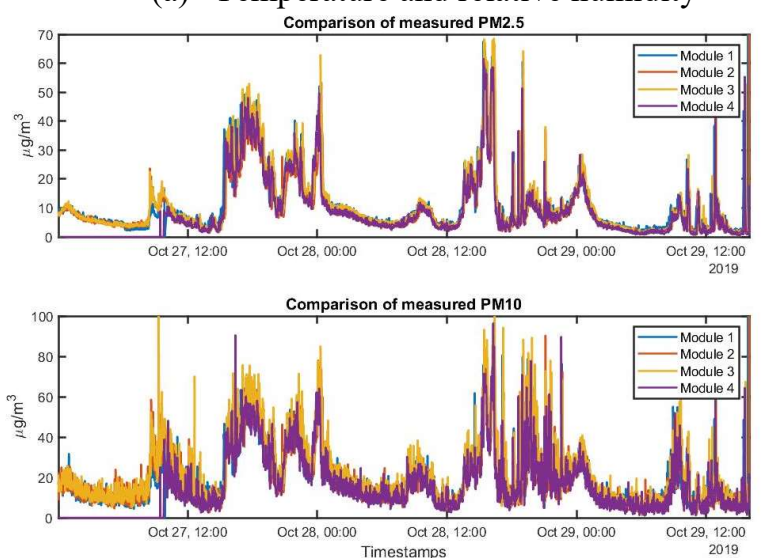

(b) Particulate matters (PM2.5 and PM10)

Figure 5. Profiles of environmental variables measured from the low-cost sensor mote

The correlations from the collocated sensors are depicted for temperature in Figure 6 typically for three modules MD1, MD2 and MD3. The results show that sensors are highly linearly-correlated with Pearson's coefficient $r>0.9$. From this analysis, we could also identify a sensor module which might have some issues during measuring data. Such a problem may be caused by inappropriate calibrations, misreading of the MCU, being located close to a noise source, or weather volatility.

Table 5 shows deviations in terms of MAE and RMSE of the inexpensive sensors collocated in the same spot. Therein, the highest MAE and RMSE can be observed for PM records, which remain an important component in monitoring of construction sites, particularly subject to a difficult environment. The dissimilarity could stem from calibrated issues that would require some benchmarking with authority stations for comparison and verification.



Figure 6. Correlations of temperature by three modules

Table 5. Calculated RMSE and MAE of the sensors

\begin{tabular}{ccc}
\hline Types of sensors & $\begin{array}{c}\text { Ranges of } \\
\text { RMSE }\end{array}$ & $\begin{array}{c}\text { Ranges of } \\
\text { MAE }\end{array}$ \\
\hline Temperature $\left({ }^{\circ} \mathrm{C}\right)$ & $0.48-0.72$ & $0.37-0.69$ \\
RH $(\%)$ & $1.72-3.20$ & $1.23-2.1$ \\
PM2.5 $\left(\mu \mathrm{g} / \mathrm{m}^{3}\right)$ & $8.37-11.2$ & $5.22-7.22$ \\
PM10 $\left(\mu \mathrm{g} / \mathrm{m}^{3}\right)$ & $12.57-15.34$ & $8.38-9.14$ \\
Soil moisture $(\%)$ & $6.13-6.55$ & $4.60-8.83$ \\
Pressure (mbar) & $0.51-0.99$ & $0.36-1.12$ \\
\hline
\end{tabular}

\subsection{Imputation for missing data}

Missing data remain an issue that may cause misinterpretation or bias upon using low-cost WSN. Unlike other studies where missing values are imputed in the offline processing phases, here we propose the stochastic regression scheme that can be integrated in the embedded program to predict the lost data by considering the correlation of the previous data collected from all colocated sensors. The gateway motes could estimate online after receiving data from the sensor motes. The benefit from a co-located sensing system is that the data from well-operated modules can be used as the references without concerning the temporal and spatial distribution of other sensor motes as in [14]. 
Let $P_{k+1}^{j}$ be the imputed value from module $j^{\text {th }}(j=1$, $2,3$ or 4$)$ missing information at the index $(k+1)$. During the operation, if device $j^{\text {th }}$ sends no data to the gateway, the gateway will poll $d$ previous values received from all four sensors (assuming all previous $d$ values are valid). Given known values of redundant data $\left(P_{k+1}^{i}\right)$, the imputation is given by

$$
P_{k+1}^{j}=\sum_{k-d}^{k}\left(w_{i j} \cdot P_{k+1}^{i}\right)+\operatorname{rand}\left(s t d^{j}\right),
$$

where the weights $w_{i j}$ are calculated by

$$
w_{i j}=\frac{r_{i j}}{\sum r_{i j}},
$$

in which $r_{i j}$ are the correlation coefficients of module $i^{\text {th }}$ and module $j^{\text {th }}(i \neq j$, e.g. if $j=3$ then $i=1,2,4)$, obtained in the range of indices from $(k-d)$ to $k$. A random number, $\operatorname{rand}($.$) , in dependence of the standard$ deviation of sensor $j^{\text {th }}\left(s t d^{j}\right)$, is added to ensure the estimated data being close to the actual measured data but not overfitting.

To validate the imputation method, we assume 1000 datapoints of temperature and $\mathrm{RH}$ in module MD3 are missed during the service time, while the others (modules 1,2 and 4) still operate well with full transceiving of data packets. The selected polling threshold is $d=20$ (i.e. 20 previous samples of the missing point are used to identify coefficients and weights). Figure 7 shows the imputation results for missing values of temperature and $\mathrm{RH}$. The estimated and the actual data relatively fit with small residuals. The RMSE of those data are 0.684 (Celsius degree) for temperature and 2.84 for $\mathrm{RH}$. Therefore, the proposed technique is promising in improving reliability of the monitoring system for the construction site.
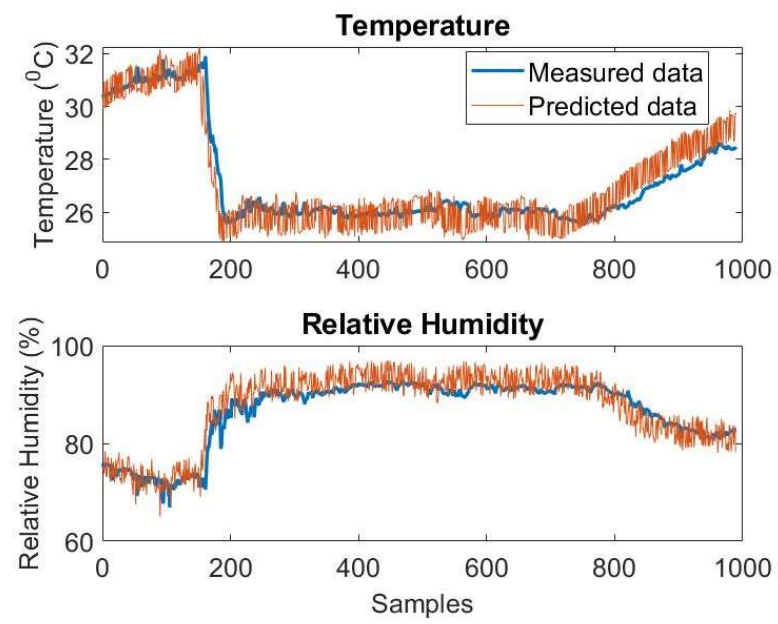

Figure 7. Estimated missing data of temperature and $\mathrm{RH}$ sensors from imputation.

\section{Field test and results: residential construction site}

The proposed dependable scheme is now adopted for monitoring the emissions of a construction site at coordinates $33^{\circ} 49^{\prime} 11^{\prime \prime} \mathrm{S}, 151^{\circ} 4^{\prime} 38^{\prime \prime} \mathrm{E}$. The low-cost sensor motes are fixed on the electrical poles at the height of 3 meters above the ground to assure a proper coverage without damage risk from construction activities.

There were 15 devices denoted as T1 to T15 installed over the whole area of approximately $1 \mathrm{~km}^{2}$. The construction site was monitored by co-located sensor motes $\mathrm{T} 1$ to $\mathrm{T} 8$, while the surrounding residential zones were monitored by $\mathrm{T} 9$ to $\mathrm{T} 15$, as depicted in Figure 8 .

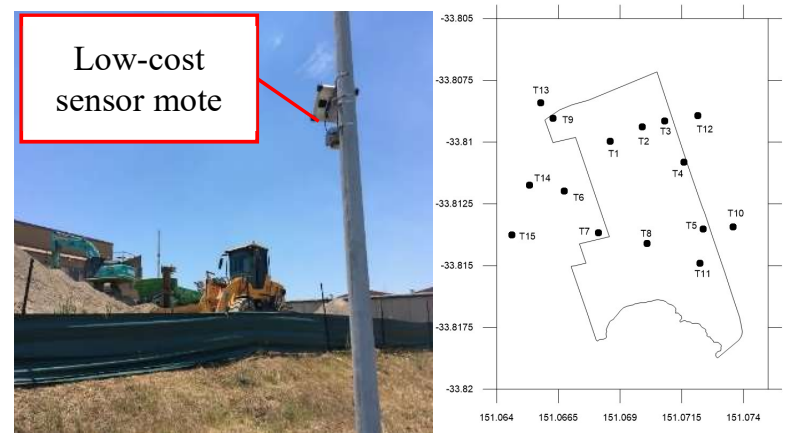

Figure 8. Low-cost sensing devices installed over the monitoring area

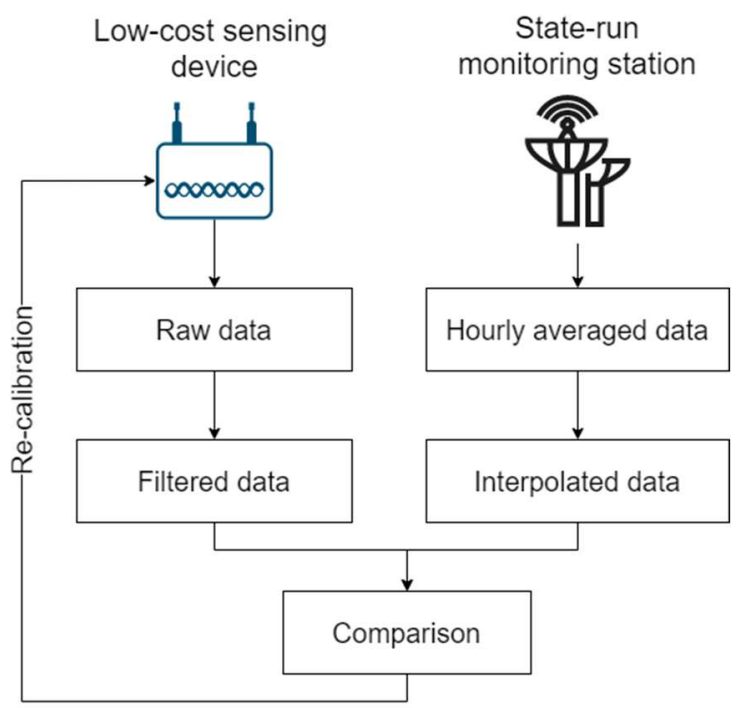

Figure 9. The iterative process of data processing of low-cost IoT-enabled monitoring system

Figure 9 presents the process of data interpolation and for long-term monitoring operations. Therein, two streams are considered: (1) data from a nearby state-run monitoring station, extracted for referencing; (2) data 
measured by the developed system on the construction site. Both data are processed prior to comparison, and the differences will be analyzed and fed back to the devices for re-calibrating to reduce any issues in association (e.g. time drift, gain and bias, etc.).

\subsection{Processing measured data}

The raw data are collected from the devices wirelessly through the Thingspeak server. The surveying period covered 65 days (from $15^{\text {th }}$ November 2019 to $19^{\text {th }}$ January 2020), with 6161 samples/device for each sensor (temperature, RH, PM2.5, PM10 and battery voltage). For the scope of this paper, only data of fine particle concentrations (PM2.5) are used for evaluating the performance of the wireless sensing system. The lowcost sensors collected data continuously 24 hours per day with a sampling period of 15 minutes to ensure stable operation, network connection, and battery lifetime. The raw data were affected by multiple noises causing imperfect recording of monitored parameters, for which anomalous values were treated by a moving average filter. Figure 10 presents the graphs before and after processing. It can be observed of the contribution from construction emissions to the rise of PM2.5 level when resuming the onsite work after the New Year break, while the high concentration of the fine particle dust reflected the impact of bushfires at the time.

\subsection{Interpolating reference data}

For validation of information gathered by low-cost devices, the measured data are compared with the data recorded by state monitoring stations, managed by the Department of Planning, Industry and Environments (DPIE) of the NSW Government [15]. Here, the closest state-run monitoring station is about $7 \mathrm{~km}$ to the site. In order to match with the time scale of the measured data, the DPIE records are resampled, with a cubic interpolation method, from 1920 to 6161 samples over the studied period.

Figure 11 shows the comparison between the two datasets after processing, which display a good fit with a high correlation as presented in Figure 12 despite some small phase shifts between two temporal profiles. This could be due to the spatial difference between the two locations with a systemic delay in DPIE reporting as well as the effects from other meteorological parameters.

The collected data from all 15 low-cost sensor motes are visualized in $3 \mathrm{D}$ for the spatial distribution by Surfer ${ }^{\mathbb{B}}$ v.11 and MATLAB $^{\circledR}$ v.2020a software for further evaluation the influence of construction activities to the air pollution over the surrounding residential area, as depicted in Figure 13, with data being obtained on Jan $8^{\text {th }}$ 2020. Therein, it can be observed of some higher concentration of PM2.5 (over $90 \mu \mathrm{g} / \mathrm{m}^{3}$ ) at the construction site, whereas the lower levels were in the residential area albeit still above the national threshold of $25 \mu \mathrm{g} / \mathrm{m}^{3}$ [16]. The reason was attributed to the prolonged impact of bushfires in the state [17].

\section{Conclusion}

This paper has presented the development of effective wireless sensing networks for reliable monitoring of construction sites using IoT-enabled dependable colocated low-cost sensor motes. The availability and resilience of the proposed system is assured by the saving-energy modes of hardware architecture, DEC framework and the redundancy of stand-by co-located sensing modules. Extensive field tests have been conducted on construction sites at different environments to validate and verify the advantages of the system implementation and its meritorious attributes. The proposed monitoring systems, coupled with various data processing techniques and the developed imputation algorithm, have resulted in significant improvement of the monitoring performance in terms of accuracy and dependability. Our future work will focus on the incorporation of learning schemes in order to cope with site monitoring in challenging conditions of abrupt changes in a volatile environment for accurate assessment of micro-climate conditions.



Figure 10. Raw and processed data from a lowcost sensor mote for PM2.5

\section{Acknowledgement}

This work is supported by Project 1032959 PRO197375 at University of Technology Sydney, and by the Vingroup Science and Technology Scholarship (VSTS) Program for Overseas Study for Master's and Doctoral Degrees. The VSTS Program is managed by VinUniversity and sponsored by Vingroup. 




Figure 11. Comparison of measured (red) and reference data (blue)

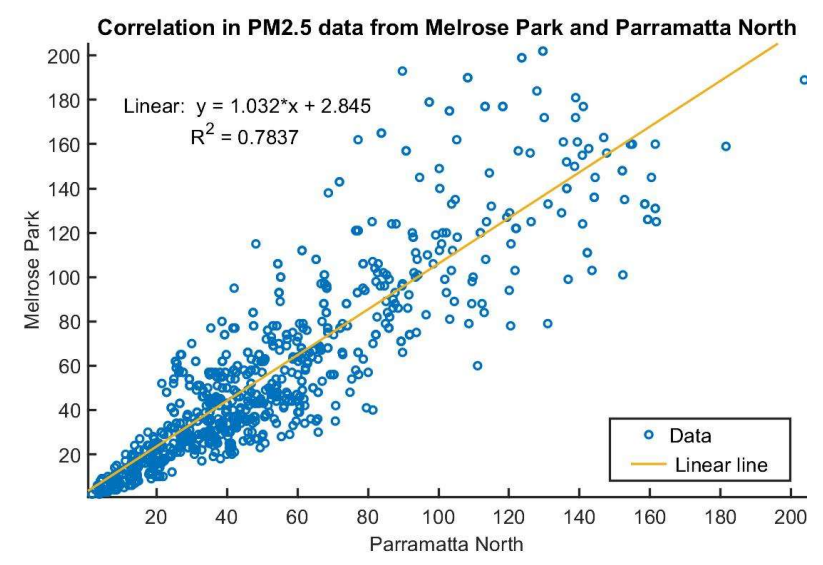

Figure 12. Correlation of two datasets by a sensor mote and by a state-run monitoring station

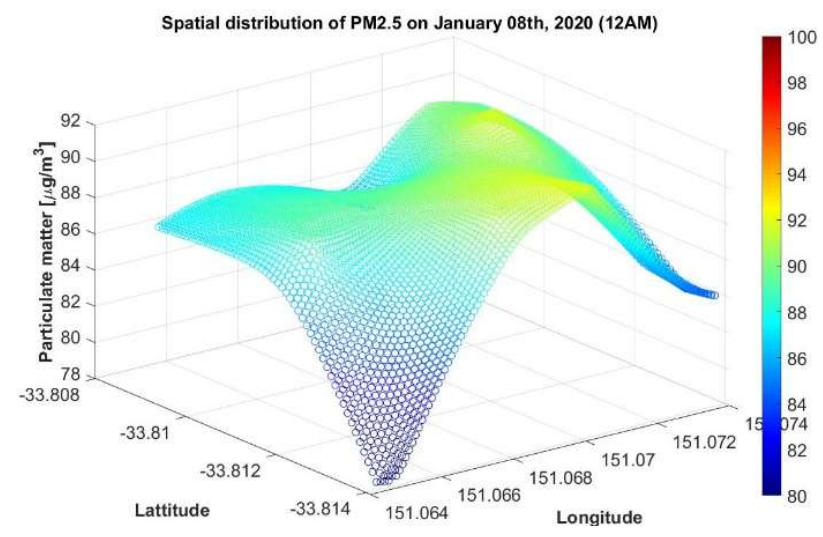

Figure 13. Spatial distribution of 15 sensor motes over the whole studied area for PM2.5

\section{References}

[1] Carotenuto F., Brilli L., Gioli B., Gualtieri G., Vagnoli C., Mazzola M., and Zaldei A. Long-Term Performance Assessment of Low-Cost Atmospheric Sensors in the Arctic Environment. Sensors, 20(7), 2020. DOI: 10.3390/s20071919.

[2] $\mathrm{Xu}$ G., Shen W., and Wang X. Applications of wireless sensor networks in marine environment monitoring: a survey. Sensors, 14(9), 16932-16954, 2014. DOI: https://doi.org/10.3390/s140916932.

[3] Reja V.K. and Varghese K. Impact of 5G Technology on IoT Applications in Construction Project Management. In Proceedings of the 36th ISARC, Banff, Alberta, Canada, pp. 209-217, 2019. DOI: https://doi.org/10.22260/ISARC2019/0029.

[4] Tagliabue L. C. and Ciribini A. L. C. A BIM-Based IoT Approach to the Construction Site Management. Journal of Structural Integrity and Maintenance, 3(4), 254-261, 2018.2 DOI: https://doi.org/10.6092/issn.2036-1602/8827

[5] Ha Q.P., Yen L. and Balaguer C. Robotic Autonomous Systems for Earthmoving in Military Applications. Automation in Construction, 107, 2019. DOI: 10.1016/j.autcon.2019.102934.

[6] Andrade E. and Nogueira B. Dependability evaluation of a disaster recovery solution for IoT infrastructures. J. of Supercomputing, 76(3), 18281849, 2020. DOI: 10.1007/s11227-018-2290-0.

[7] Bruneo D., Puliafito A. and Scarpa M. Dependability evaluation of Wireless Sensor Networks: Redundancy and topological aspects. IEEE Sensors, 1827-1831, 2010. DOI: https://doi.org/10.1109/ICSENS.2010.5690005.

[8] Silva I. Leandro R., Macedo D. and Guedes L. A dependability evaluation tool for the Internet of Things. Computers and Electrical Engineering, 39(7), 2005-2018, 2013. DOI: https://doi.org/10.1016/j.compeleceng.2013.04.021.

[9] Ha Q.P. and Phung M.D. IoT-enabled dependable control for solar energy harvesting in smart buildings. IET Smart Cities, vol. 1, no. 2, pp. 61-70, 2019. DOI: 10.1049/iet-smc.2019.0052.

[10] Espressif Systems. ESP32 Series the Datasheet, version $3.4, \quad 2020 . \quad$ Online: https://www.espressif.com/sites/default/files/docu mentation/esp32 datasheet en.pdf, Accessed: 12/05/2020.

[11] Panasonic. Datasheet of Lithium Ion NCR18650B. Online: $\quad$ https://www.batteryspace.com/prodspecs/NCR18650B.pdf. Accessed: 01/05/2020.

[12] Tran T., Ha Q.P. and Hunjet R. "Reliability Enhancement with Dependable Model Predictive Control," ISA Transactions, https://doi.org/10.1016/j.isatra.2020.06.027, online 6 July 2020. 
[13] Heumann C., Schomaker M. and Shalabh S. Introduction to Statistics and Data Analysis With Exercises, Solutions and Applications in $R$, Springer, Cham, ISBN 978-3-319-46162-5, 2017. DOI: https://doi.org/10.1007/978-3-319-46162-5.

[14] Li Y. and Parker L. A spatial-temporal imputation technique for classification with missing data in a wireless sensor network. In Proceeding of IEEE/RSJ International Conference on Intelligent Robots and Systems, 3272-3279, 2008. DOI: https://doi.org/10.1109/IROS.2008.4650774.

[15] New South Wale Government - Planning Industry and Environment. Online: https://www.dpie.nsw.gov.au/air-quality/searchfor-and-download-air-quality-data, $\quad$ Accessed: $24 / 04 / 2020$

[16] Australia - State of the Environment. National Air Quality Standard. Online: https://soe.environment.gov.au/theme/ambient-airquality/topic/2016/national-air-quality-standards, Accessed: 21/04/2020.

[17] Ha Q.P., Nguyen H.A.D., and Metia S. Construction Site Impact on Urban Air Quality using Low-cost Sensing Networks. In Melrose Park: Smart Planning for Climate Responsive Neighbourhoods, Report PRO19-7375. 